Aus der Abteilung für Hautkranke der Graf Apponyi-Poliklinik in Budapest. (Vorstand: Prof. L. Török.)

\title{
Verhinderung von Intoxikationserscheinungen (Fieber, Haufentzündung) nach Neosalvarsaninjektionen durch gleichzeitige Verabreichung von Calcium bromatum und Calcium chloratum.
}

Von Dr. Desider Kenedy, Assistent.

In Nr. 7 berichtet E. Pula y über die Beseitigung unangenehmer Nebenerscheinungen der Salvarsantherapie bei Syphiliskranken mit Affektionen des Nervensystems und innerer Organe durch SalvarsanKalzium-Mischspritzen. Dies gibt mir Veranlassung zu nachstehender kurzer Mitteilung.

Im Vorjahre behandelte ich eine Patientin, bei der schon nach geringer Dosis $(0,15$ bis 0,30$)$ Neosalvarsan hochgradiges Oedem und diffuse Hautentzündung des Gesichtes und der Extremitäten auftraten, von heftiger allgemeiner Reaktion begleitet $\left(40^{\circ}\right.$ Fieber), soda $B$ ich gezwungen war, den weiteren Gebrauch von Salvarsan zu unterbrechen. Auf Grund der Erfahrungen, die wir auf unserer Abteilung bei der Behandlung urtikarieller Anfälle mit Optokalcil machten (eine hypertonische kalzinierte Calcium bromatum purissimum-Lösung) worüber wir demnächst ausführlich berichten werden -, gab ich der Patientin Kalzium, und zwar vorerst sofort an die Salvarsaninjektion anschlieBend, später, nachdem ich mich von der Unschädlichkeit des in Optokalcil gelösten Salvarsans überzeugt hatte, in diesem gelöst. Seither vertrug die Kranke Salvarsan ohne Beschwerden.

Die Krankengeschichte der Patientin ist folgende: Hrau P.E., 30 Jahre alt; ausgebreitetes papulös-krustöses syphilitisches Exanthem; bis zum heutigen Tage erhielt sie $30 \mathrm{Hg}$-Spritzen von ihrem Hausarzt, ohne daB sich die Hauterscheinungen rückgebildet hätten.

23. V. 1921 - 0,15 Neosalvarsan. Abends Kopfschmerzen, Patientin fühlt sich fieberisch.

28. V. - 0,30 Neosalvarsan. Abends Schüttelfrost (Temperatur $\left.40^{\circ}\right)$. Extremitäten, Gesicht stark ödematös, Augen verschwollen. Die Haut des Gesichtes und der Extremitäten, hauptsächlich aber die der oberen Extremitäten, ist diffas lebhaft rot und faBt sich heiß an. Im Urin kein Eiweiß vorhanden. Am nächsten Tag ist Patientin fieberfrei, "am dritten Tag haben sich Oedem und Dermatitis zurückgebildet.

9. VI. - 0,15 Neosalvarsan. Abends neuerdings $40^{\circ}$ Fieber, Oedem, Dermatitis. Dieser Anfall dauert auch nur drei Tage. Da die Patientih stark abgenommen hat und die syphilitischen Hauterscheinungen vollständig abgeheilt waren, unterbrach ich die Salvarsanbehandlung.

Am 29. X. 1921 meldete sich die Patientin wieder. Ich versuche 0,15 Neosalvarsan wieder. Abends vollständig gleicher Krankheitszustand. Fieber, Oedem Dermatitis.

6 . XI. - 0,15 Neosalvarsan und sofort anschliebend $10 \mathrm{ccm}$ Uptokalcil ( $M$ a rberger). Es zeigte sich weder Fieber, noch Oedem oder Dermatitis. Die Patientin fühlte sich vollständig wohl.

11. XI. - 0,30 Neosalvarsan in $10 \mathrm{ccm}$ Optokalcil gelöst.

Seither erhielt Patientin 16 Salvarsanspritzen à 0,30 in Optokalcil gelöst. Es zeigten sich keinerlei unangenehme Erscheinungen.

Später wandte ich statt Optokalcil eine $10 \%$ ige Calcium chloratumLösung an, mit ähnlichem Erfolge. 